Kansas State University Libraries

New Prairie Press

\title{
A FERTILIZER-RATE EXPERIMENT INVOLVING YOUNG CITRUS TREES: DOES MORE FERTILIZER MEAN HIGHER PRODUCING TREES?
}

John A. Cornell

Follow this and additional works at: https://newprairiepress.org/agstatconference

Part of the Agriculture Commons, and the Applied Statistics Commons

\section{(c) (1) $\Theta(9$}

This work is licensed under a Creative Commons Attribution-Noncommercial-No Derivative Works 4.0 License.

\section{Recommended Citation}

Cornell, John A. (2000). "A FERTILIZER-RATE EXPERIMENT INVOLVING YOUNG CITRUS TREES: DOES MORE FERTILIZER MEAN HIGHER PRODUCING TREES?," Conference on Applied Statistics in Agriculture. https://doi.org/10.4148/2475-7772.1236

This is brought to you for free and open access by the Conferences at New Prairie Press. It has been accepted for inclusion in Conference on Applied Statistics in Agriculture by an authorized administrator of New Prairie Press. For more information, please contact cads@k-state.edu. 


\title{
A FERTILIZER-RATE EXPERIMENT INVOLVING YOUNG CITRUS TREES: DOES MORE FERTILIZER MEAN HIGHER PRODUCING TREES?
}

\author{
John A. Cornell \\ Agricultural Experiment Station \\ and Department of Statistics \\ University of Florida \\ Gainesville, Florida 32611
}

\begin{abstract}
Citrus growers are interested in making money. So, the most common practice among growers is to push young trees into early production by the application of high amounts (rates) of fertilizer. This practice can lead to disaster in terms of tree formation (canopy shape) and production stress. In contrast, when the applied fertilizer approaches both the optimum rate and the optimum N-P-K-Ca ratio for citrus, then the trees are more uniform in size and with compact canopies and the incidence of decline is less. Cordieropolis station in São Paula, Brazil, is the site of a large 3-component by 3 rates fertilizer experiment on young citrus (orange) trees. We shall present the statistical aspects (design, model, and the method of data analysis) of the experiment along with the surprising results obtained thus far.
\end{abstract}

\section{Introduction}

Mixture experiments are performed in many areas of agricultural research. In a mixture experiment, two or more ingredients are mixed or blended together in varying proportions to form some end product. Quality characteristics of the end product are recorded for each blend in an effort to see if the characteristics change from one blend to the next. The actual experiment consists of varying the ingredient proportions and studying the changes that occur in the measured response or responses assumed to be functionally related to ingredient composition. The controllable variables are nonnegative proportionate amounts of the mixture in which the proportions are by volume, weight, or mole fraction. In a q-ingredient (or q-component) mixture in which $\mathrm{x}_{\mathrm{i}}$ represents the proportion of the ith component present in the mixture,

$$
0 \leq \mathrm{x}_{\mathrm{i}} \leq 1, \quad \mathrm{i}=1,2, \ldots, \mathrm{q}
$$

and

$$
\sum_{i=1}^{q} x_{i}=1
$$

The composition space of the q components, by virtue of the restrictions (1) and (2), takes the form of a (q-1)-dimensional simplex. When additional constraints are imposed on the component proportions in the form of lower and upper bounds, say $L_{i}$ and $U_{i}$, respectively, 


$$
0<\mathrm{L}_{\mathrm{i}} \leq \mathrm{x}_{\mathrm{i}} \leq \mathrm{U}_{\mathrm{i}}<1, \quad \mathrm{i}=1,2, \ldots, \mathrm{q},
$$

the resulting region, called a constrained region, is a subregion of the simplex whose shape is not that of a simplex. As an example, suppose we have three juice sources, watermelon $\left(\mathrm{x}_{1}\right)$, pineapple $\left(\mathrm{x}_{2}\right)$, and orange $\left(\mathrm{x}_{3}\right)$ in which to make a fruit punch. Suppose further that we wish to restrict the punch blends to consisting of at least $30 \%$ watermelon but not more than $60 \%$ watermelon, at least $5 \%$ pineapple but not more than $45 \%$ pineapple and orange juice to comprise between $20 \%$ and $60 \%$ of the punch. Proportion-wise, these restrictions are listed as

$$
\begin{aligned}
& 0.30 \leq \mathrm{x}_{1} \leq 0.60 \\
& 0.05 \leq \mathrm{x}_{2} \leq 0.45 \\
& 0.20 \leq \mathrm{x}_{3} \leq 0.60
\end{aligned}
$$

The constrained region defined by the constraints in (4) is shown in Figure 1.

In mixture experiments, the focus of attention is on the blending properties of the components in the mixture. These blending properties are called linear and nonlinear blending depending on whether the value of the response changes linearly or nonlinearly when changing the composition of the ingredients in the blend. The blending properties of the components are determined by fitting special types of mixture models to data collected from the various mixture blends used. The most popular types of mixture models are the canonical forms of polynomials introduced by Scheffé (1958). For $\mathrm{q}=3$ components whose proportions are denoted by $\mathrm{x}_{1}, \mathrm{x}_{2}$ and $\mathrm{x}_{3}$, the forms of the linear, quadratic, and special cubic mixture models are

Linear:

$$
\text { Response }=\beta_{1} x_{1}+\beta_{2} x_{2}+\beta_{3} x_{3}+\varepsilon
$$

Quadratic:

$$
\text { Response }=\beta_{1} x_{1}+\beta_{2} x_{2}+\beta_{3} x_{3}+\beta_{12} x_{1} x_{2}+\beta_{13} x_{1} x_{3}+\beta_{23} x_{2} x_{3}+\varepsilon
$$

Special Cubic:

$$
\text { Response }=\beta_{1} x_{1}+\beta_{2} x_{2}+\beta_{3} x_{3}+\beta_{12} x_{1} x_{2}+\beta_{13} x_{1} x_{3}+\beta_{23} x_{2} x_{3}+\beta_{123} x_{1} x_{2} x_{3}+\varepsilon
$$

In each of the models (5), (6), and (7) above, the quantities $\beta_{\mathrm{i}}, \beta_{\mathrm{ij}}$, and $\beta_{123}$ are unknown parameters or coefficients whose values are estimated from the experimental data and $\varepsilon$ is a random error term. For additional discussion on Scheffé and other mixture model forms, see Cornell (1990).

\section{Mixture-amount Experiments}

A mixture-amount (M-A) experiment is a mixture experiment that is performed at two or more levels of total amount. In a M-A experiment, the response is expected to depend on the relative proportions of the ingredients present in the mixture and on the amount of the mixture as well. An example of a M-A experiment that we shall discuss more fully in the next section is a 3-component (Nitrogen- $\mathrm{P}_{2} \mathrm{O}_{5}-\mathrm{K}_{2} \mathrm{O}$ ) fertilizer experiment where each of nine fertilizer blends chosen for study is 
applied at three rates (low, medium, and high). Designs for M-A experiments are discussed in Piepel and Cornell (1987) and in Cornell (1990), Chapter 7.

The form of the combined model to be fitted to data collected in a M-A experiment depends on how the effect of amount is defined. Consider for example an experiment where three levels of amount are to be studied. Changing the amount of the mixture can affect the response in one of two ways. It can affect the response value only and not affect the blending properties of the mixture components, or, it can affect the value of the response by affecting the blending properties of the components. In the case of affecting the value of the response only, the effect of amount is said to be additive and the M-A model is expressed as

$$
\begin{aligned}
\text { Response } & =\{\text { mixture model }\}+\alpha_{1} \mathrm{~A}+\varepsilon \quad \text { (Linear effect of amount) } \\
& =\{\text { mixture model }\}+\alpha_{1} \mathrm{~A}+\alpha_{2} \mathrm{~A}^{2}+\varepsilon \quad \text { (Quadratic effect of amount) }
\end{aligned}
$$

where $\mathrm{A}$ is a coded variable for the amount of the mixture. On the other hand, when changing the level of amount affects the blending properties of the components, the blending properties are said to interact with the amount effect and the model is expressed as

$$
\begin{aligned}
\text { Response } & =\{\text { mixture model }\}+\{\text { mixture model }\} \mathrm{A}+\varepsilon \\
& =\{\text { mixture model }\}+\{\text { mixture model }\} \mathrm{A}+\{\text { mixture model }\} \mathrm{A}^{2}+\varepsilon
\end{aligned}
$$

For additional discussion on the fitting of M-A models, see Cornell (1990), Chapter 7.

\section{The Mixture-Amount Experiment in São Paulo.}

Cordieropolis station in São Paulo, Brazil is the site of a large fertilizer-rate experiment currently underway on 3-year old citrus trees. At the initial planning stages of the experiment, mixes or blends of four fertilizer ingredients, nitrogen $(\mathrm{N})$, phosphorus $\left(\mathrm{P}_{2} \mathrm{O}_{5}\right.$ or $\left.\mathrm{P}\right)$, potash $\left(\mathrm{K}_{2} \mathrm{O}\right.$ or $\left.\mathrm{K}\right)$ and calcium (Ca) were considered along with three application rates, low, medium, and high. The lower and upper bound percentages for the ingredients were

$$
\begin{aligned}
30 \% & \leq \mathrm{N} \leq 60 \% \\
4 \% & \leq \mathrm{P} \leq 33 \% \\
27 \% & \leq \mathrm{K} \leq 56 \% \\
0 \% & \leq \mathrm{Ca} \leq 20 \%
\end{aligned}
$$

which when expressed as proportions of a fertilizer blend (i.e., divided by 100\%) are

$$
\begin{aligned}
0.30 & \leq \mathrm{x}_{1} \leq 0.60 \\
0.04 & \leq \mathrm{x}_{2} \leq 0.33 \\
0.27 & \leq \mathrm{x}_{3} \leq 0.56 \\
0 & \leq \mathrm{x}_{4} \leq 0.20
\end{aligned}
$$


where $\mathrm{x}_{1}, \mathrm{x}_{2}, \mathrm{x}_{3}$ and $\mathrm{x}_{4}$ represent the proportions of nitrogen, phosphorus, potash, and calcium, respectively. The constrained region (10) has 12 extreme vertices, 18 edges, and eight 2-dimensional faces and is shown in Figure 2.

To support fitting the 4-component quadratic model of the form (6), ten distinct blends are required and 15 were chosen. The fifteen different blends that were chosen by the DESIGNEXPERT software of Stat-Ease (1993) were selected from a candidate list of 50 blends and are represented by 11 of the 12 extreme vertices, midpoints of 2 of the 18 edges, one 2 -dimensional face centroid, and an interior point located very near the centroid of the region. The plan was to collect data from the fifteen distinct blends plus second replicates of five of the 15 blends for a total of twenty blends at each level of rate. The twenty data values would then be fitted by the quadratic model at each rate. Then, to the total data set of 60 values, we would fit the combined model of the form (9).

However, a change was made in the plans. Instead of four components, it was decided to simplify the experiment by focusing only on the three most important ingredients which are nitrogen $(\mathrm{N})$, phosphorus $\left(\mathrm{P}_{2} \mathrm{O}_{5}\right)$ and potash $\left(\mathrm{K}_{2} \mathrm{O}\right)$. Furthermore, at each fertilizer rate, the form of the mixture model to be fitted was expanded to include the special-cubic term $\beta_{123} \mathrm{x}_{1}{ }^{\prime} \mathrm{x}_{2}{ }^{\prime} \mathrm{x}_{3}{ }^{\prime}$ in the L-pseudocomponents

$$
\mathrm{TD}=\beta_{1} \mathrm{x}_{1}{ }^{\prime}+\beta_{2} \mathrm{x}_{2}{ }^{\prime}+\beta_{3} \mathrm{x}_{3}{ }^{\prime}+\beta_{12} \mathrm{x}_{1}{ }^{\prime} \mathrm{x}_{2}{ }^{\prime}+\beta_{13} \mathrm{x}_{1}{ }^{\prime} \mathrm{x}_{3}{ }^{\prime}+\beta_{23} \mathrm{x}_{2}{ }^{\prime} \mathrm{x}_{3}{ }^{\prime}+\beta_{123} \mathrm{x}_{1}{ }^{\prime} \mathrm{x}_{2}{ }^{\prime} \mathrm{x}_{3}{ }^{\prime}+\varepsilon
$$

where TD represents the trunk diameter of the citrus trees and $\mathrm{x}_{1}{ }^{\prime}, \mathrm{x}_{2}{ }^{\prime}$ and $\mathrm{x}_{3}{ }^{\prime}$ represent L-pseudocomponents defined as

$$
x_{1}^{\prime}=\frac{x_{1}-.30}{.39} \quad x_{2}^{\prime}=\frac{x_{2}-.04}{.39} \quad x_{3}^{\prime}=\frac{x_{3}-.27}{.39}
$$

In the denominator the quantity $.39=1-(.30+.04+.27)$ is the height of the L-pseudocomponent triangle relative to a height of unity for the original triangle. The experimental region in the Lpseudocomponents has 6 extreme vertices and 5 edges and is shown in Figure 3. Listed in Table 1 are the coordinate settings in the L-pseudocomponents along with the actual percentages of the original components $\mathrm{N}, \mathrm{P}_{2} \mathrm{O}_{5}$ and $\mathrm{K}_{2} \mathrm{O}$ of nine fertilizer blends that were applied to plots. Listed also is the average trunk diameter value of eight trees per plot in each of two replicate plots receiving the same treatment. The trunk diameter values, measured in millimeters, are listed at each of the three rates.

At the low, medium, and high rates, the coefficient estimates of the fitted special cubic models are listed in Table 2. Also listed in Table 2 are the values of $\mathrm{R}^{2}$ approx. and Mean Square Error for each of the three fitted models along with significance levels of tests performed on the individual coefficient estimates of the nonlinear blending terms when the significance level was less than 0.05. Nearly all of the nonlinear blending terms played important roles at the low and high fertilization rates but not necessarily at the medium rate. The magnitudes of the low, medium, and high rates relative to the standard rate of fertilization were low $=(1 / 3)$ standard rate, medium $=(2.5 / 3)$ standard rate, and high $=(4 / 3)$ standard rate, respectively. Contour plots of the estimated average trunk 
diameter surfaces generated by the fitted models in Table 2 at the low, medium, and high rates are shown in Figure 4.

To the total data set of $3 \times 18=54$ values, the fitted combined model in the L-pseudocomponents and coded fertilizer rate is

$$
\begin{aligned}
& \mathrm{TD}=\{\text { mixture model }\}+\{\text { mixture model }\} \boldsymbol{A}+\{\text { mixture model }\} \boldsymbol{A}^{2} \\
& =27.21 x_{1}{ }^{\prime}+27.97 x_{2}{ }^{\prime}+22.83 x_{3}{ }^{\prime}+39.07 * x_{1}{ }^{\prime} x_{2}{ }^{\prime}+55.65 * x_{1}{ }^{\prime} x_{3}{ }^{\prime}+42.99 * x_{2} x_{3}-176.53 * x_{1}{ }^{\prime} x_{2}{ }^{\prime} x_{3}{ }^{\prime} \\
& +\left\{-3.00 * x_{1}{ }^{\prime}-0.32 x_{2}{ }^{\prime}-0.60 x_{3}{ }^{\prime}+2.86 x_{1}{ }^{\prime} x_{2}{ }^{\prime}+6.45 x_{1}{ }^{\prime} x_{3}{ }^{\prime}+1.00 x_{2}{ }^{\prime} x_{3}{ }^{\prime}-11.42 x_{1}{ }^{\prime} x_{2}{ }^{\prime} x_{3}{ }^{\prime}\right\} A \\
& +\left\{-1.33 x_{1}{ }^{\prime}-2.41 * x_{2}{ }^{\prime}+0.27 x_{3}{ }^{\prime}+8.99 x_{1}{ }^{\prime} x_{2}{ }^{\prime}+0.81 x_{1}{ }^{\prime} x_{3}{ }^{\prime}+7.90 x_{2}{ }^{\prime} x_{3}{ }^{\prime}-16.04 x_{1}{ }^{\prime} x_{2}{ }^{\prime} x_{3}{ }^{\prime}\right\} A^{2}
\end{aligned}
$$

where the asterisk $(*)$ following an estimated coefficient signifies the estimate is significantly different from zero at the 0.05 level. The SAS program statements and data set along with the output of the analysis of variance table are listed in the appendix.

In the fitted model (13), $\mathrm{A}$ and $\mathrm{A}^{2}$ represent coded variables for the linear and quadratic effects of fertilizer rate or amount and take the values $\mathrm{A}=-4,-1$, and 5 and $\mathrm{A}^{2}=2,-3$, and 1 at the low, medium, and high rates, respectively. The first seven terms of (13) represent the mixture-only portion of the combined model in terms of the linear and nonlinear blending properties of the L-pseudocomponents for $\mathrm{N}, \mathrm{P}_{2} \mathrm{O}_{5}$ and $\mathrm{K}_{2} \mathrm{O}$ over the constrained region (12) when averaged over the three rates. The middle seven terms of (13) represent the linear effect of rate on the blending properties of the L-pseudocomponents while the final seven terms of (13) represent the quadratic effect of rate on the blending properties of the L-pseudocomponents.

To help us interpret the linear and nonlinear blending properties, averaged over the three rates, a contour plot of the estimated trunk diameter (TD) surface, is displayed in Figure 4. Accompanying the contour plot is a 3-dimensional surface plot of the estimated average TD surface that helps one visualize the shape of the surface. According to the two plots, the estimated trunk diameter of trees is affected positively when treated by blends located approximately at the midpoints of the three sides of the L-pseudocomponent triangle. The estimated TD is lower in value at the centroid of the triangle than at each of the mid-points of the three sides. Among the next seven terms in which the linear effect of rate is represented, the significance of - $3.00 \mathrm{x}_{1}{ }^{\prime}$ A suggests the average TD values are significantly higher at the high nitrogen blends of the region for the low and medium fertilizer rates than at the high rate. Similarly, among the final seven terms, the significance of the $-2.41 \mathrm{x}_{2}{ }^{\prime} \mathrm{A}^{2}$ term suggests the average TD values at the high potash blends are higher at the medium rate than at the low and high application rates. The contour plots in Figure 5 support these inferences.

\section{Summary of the analysis of the initial data set.}

Although we have analyzed only one set of data at this point in time, there appears to be evidence that young ( $\leq 4$ years of age) citrus trees respond more favorably to lower rates than to higher rates 
of fertilization. By responding is meant trees fertilized at the lower rate appear to have larger trunk diameters, are taller, and possess more symmetrically-shaped canopies than trees fed the same fertilizer blends at the high rate of fertilization. These results are somewhat counter or opposite to those which most extension specialists have believed traditionally over-the-years.

These initial results were available because of a large mixture-amount or mixture-rate experiment that is currently being performed in São Paulo, Brazil. A mixture-amount (MA) experiment is a mixture experiment that is performed at two or more amounts (or rates in our case) of the mixtures. Performing a MA experiment allows one to separate the effects of varying the mixture composition on the response and the effect of changing the rate of application or amount on the response.

Additional data sets on trunk diameter and other tree characteristics are expected to be received and analyzed over the next couple of years. In the meantime it is anticipated that the additional data will support our contention that,

(i) The optimal N-P-K blend will change with the age of the citrus trees and be different at the three fertilization rates.

(ii) Additional evidence of moderation in fertilizer rate is suspected and that higher does not necessarily mean better as far as the amount of fertilizer applied to citrus trees is concerned.

\section{References}

Cornell, J.A. (1990). Experiments With Mixtures. $2^{\text {nd }}$ Edition. John Wiley and Sons. New York.

Cornell, J.A. and J.M. Harrison (1999). Models and Designs for Experiments With Mixtures: Part

II - Exploring a Subregion of the Simplex and the Inclusion of Other Factors in Mixture Experiments. Technical Bulletin 899, Florida Agricultural Experiment Station, IFAS, University of Florida, Gainesville, FL.

Piepel, G.F. and J.A. Cornell (1987). Designs for Mixture-Amount Experiments. Journal of Quality Technology, 19, 11-28.

Scheffé, H. (1958). Experiments With Mixtures. Journal of the Royal Statistical Society, B, 20, 344-360.

Stat-Ease (1993). DESIGN-EXPERT Software for Response Surface Methodology and Mixture Experiments. Version 4.0, Stat-Ease, Inc., Minneapolis, Minnesota. 
Table 1. Average trunk diameter of eight trees in two replicates of each of the nine blends. Listed also are the coordinates (\%) of the original and L-pseudocomponents.

\begin{tabular}{cccc|ccc|ccc} 
& \multicolumn{4}{c}{$\%$} & & \multicolumn{2}{|c|}{ L-pseudocomponents } & \multicolumn{3}{|c}{ Trunk Diameter (mm) } \\
Blend No. & Nit. & $\mathrm{P}_{2} \mathrm{O}_{5}$ & $\mathrm{~K}_{2} \mathrm{O}$ & $x_{1}{ }^{\prime}$ & $x_{2}{ }^{\prime}$ & $x_{3}{ }^{\prime}$ & Low & Medium & High \\
\hline 1. & 52 & 16 & 32 & .564 & .308 & .128 & $40.1,40.6$ & $37.8,35.0$ & $31.6,30.4$ \\
2. & 56 & 4 & 40 & .667 & 0 & .333 & $38.4,41.1$ & $41.5,39.7$ & $32.1,33.4$ \\
3. & 46 & 8 & 46 & .410 & .103 & .487 & $37.7,38.9$ & $37.0,34.6$ & $37.9,35.6$ \\
4. & 40 & 4 & 56 & .256 & 0 & .744 & $33.9,34.6$ & $35.6,35.3$ & $34.9,33.5$ \\
5. & 30 & 20 & 50 & 0 & .410 & .590 & $39.3,38.1$ & $30.9,31.5$ & $34.3,36.5$ \\
6. & 30 & 35 & 35 & 0 & .795 & .205 & $33.8,32.9$ & $38.0,37.1$ & $31.2,33.7$ \\
7. & 36 & 36 & 28 & .154 & .821 & .025 & $34.0,32.4$ & $34.6,34.3$ & $30.7,30.6$ \\
8. & 45 & 28 & 27 & .385 & .615 & 0 & $41.2,38.1$ & $38.9,37.0$ & $33.0,33.6$ \\
9. & 42 & 18 & 40 & .308 & .359 & .333 & $37.0,36.8$ & $34.3,36.1$ & $31.4,29.4$
\end{tabular}


Table 2. Fitted special cubic mixture models and summary statistics for the trunk diameter of citrus trees at the low, medium, and high rates of fertilization.

Coefficient Estimates

\begin{tabular}{lcccccc} 
Model Term & Low & $\operatorname{Pr}>\mathrm{T}$ & Medium & $\operatorname{Pr}>\mathrm{T}$ & High & $\operatorname{Pr}>\mathrm{T}$ \\
\hline$\beta_{1} \mathrm{x}_{1}$ & 36.53 & & 34.20 & & 10.88 & \\
$\beta_{2} \mathrm{x}_{2}$ & 24.41 & & 35.51 & & 23.97 & \\
$\beta_{3} \mathrm{x}_{3}$ & 25.76 & & 22.61 & & 20.13 & \\
$\beta_{12} \mathrm{x}_{1} \mathrm{x}_{2}$ & 45.64 & .0141 & 9.24 & $\mathrm{~ns}$ & 62.34 & .0216 \\
$\beta_{13} \mathrm{x}_{1} \mathrm{x}_{3}$ & 31.47 & $\mathrm{~ns}$ & 46.78 & $\mathrm{~ns}$ & 88.69 & .0299 \\
$\beta_{23} \mathrm{x}_{2} \mathrm{x}_{3}$ & 54.78 & .0028 & 18.27 & $\mathrm{~ns}$ & 55.91 & .0234 \\
$\beta_{123} \mathrm{x}_{1} \mathrm{x}_{2} \mathrm{x}_{3}$ & -162.93 & .0129 & -116.98 & $\mathrm{~ns}$ & -249.67 & .0110 \\
$\mathrm{R}_{\text {approx. }}^{2}$ & 0.9001 & & 0.7126 & & 0.6437 & \\
$\mathrm{MSE}$ & 1.30 & & 3.14 & & 2.88 &
\end{tabular}




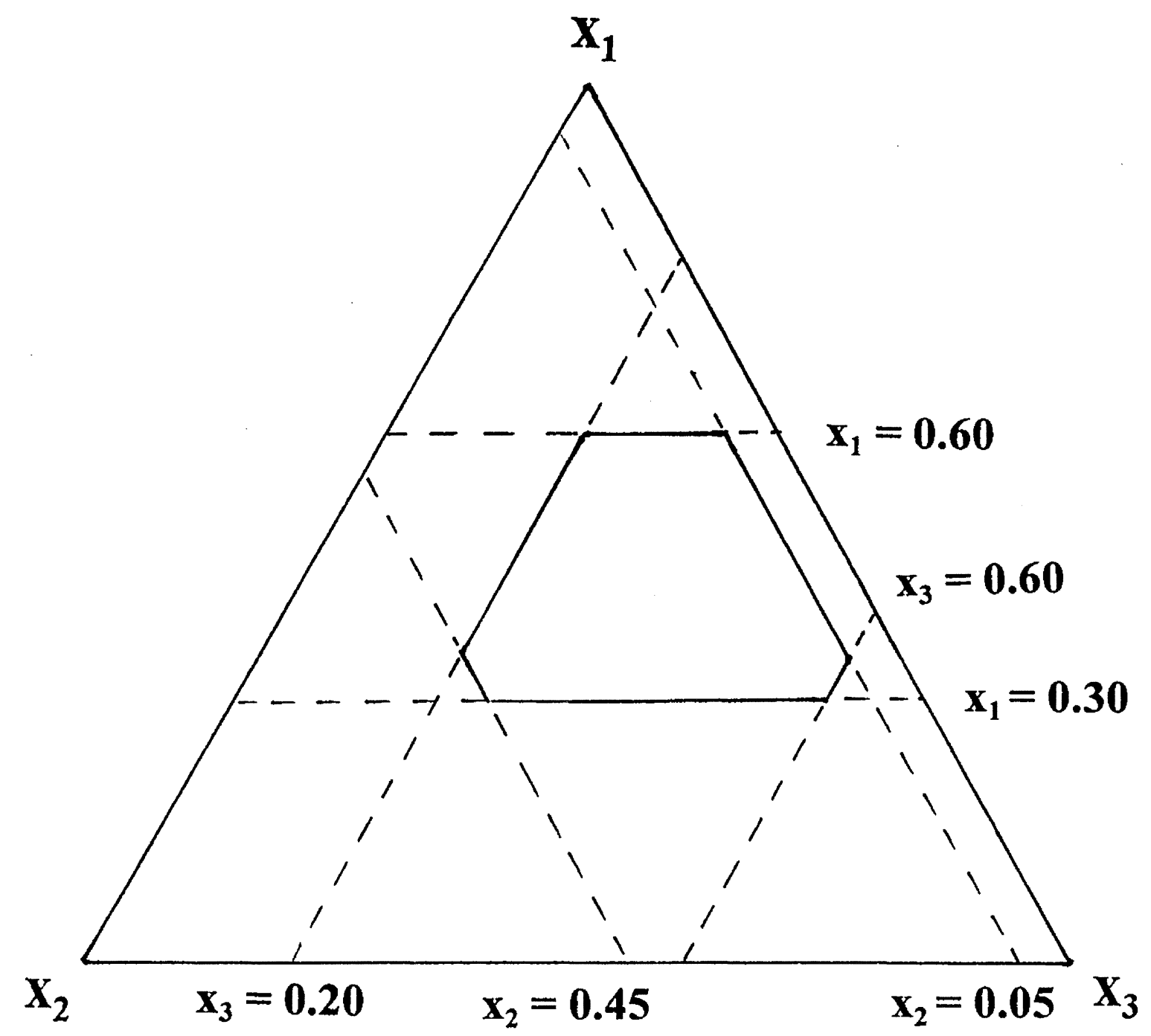

Figure 1. The constrained region defined by the constraints. 


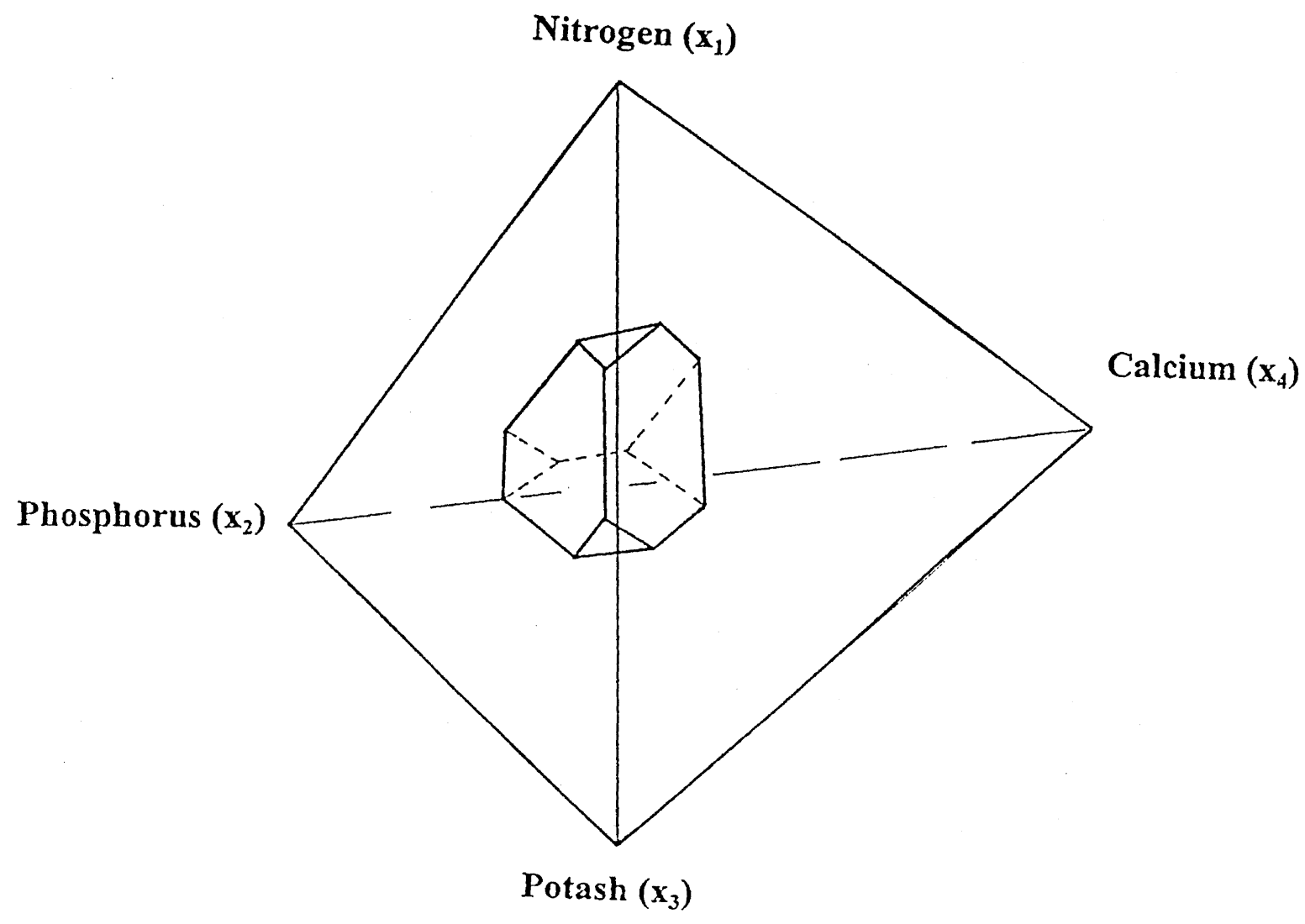

Figure 2. The 4-component constrained region defined by the constraints (10). 


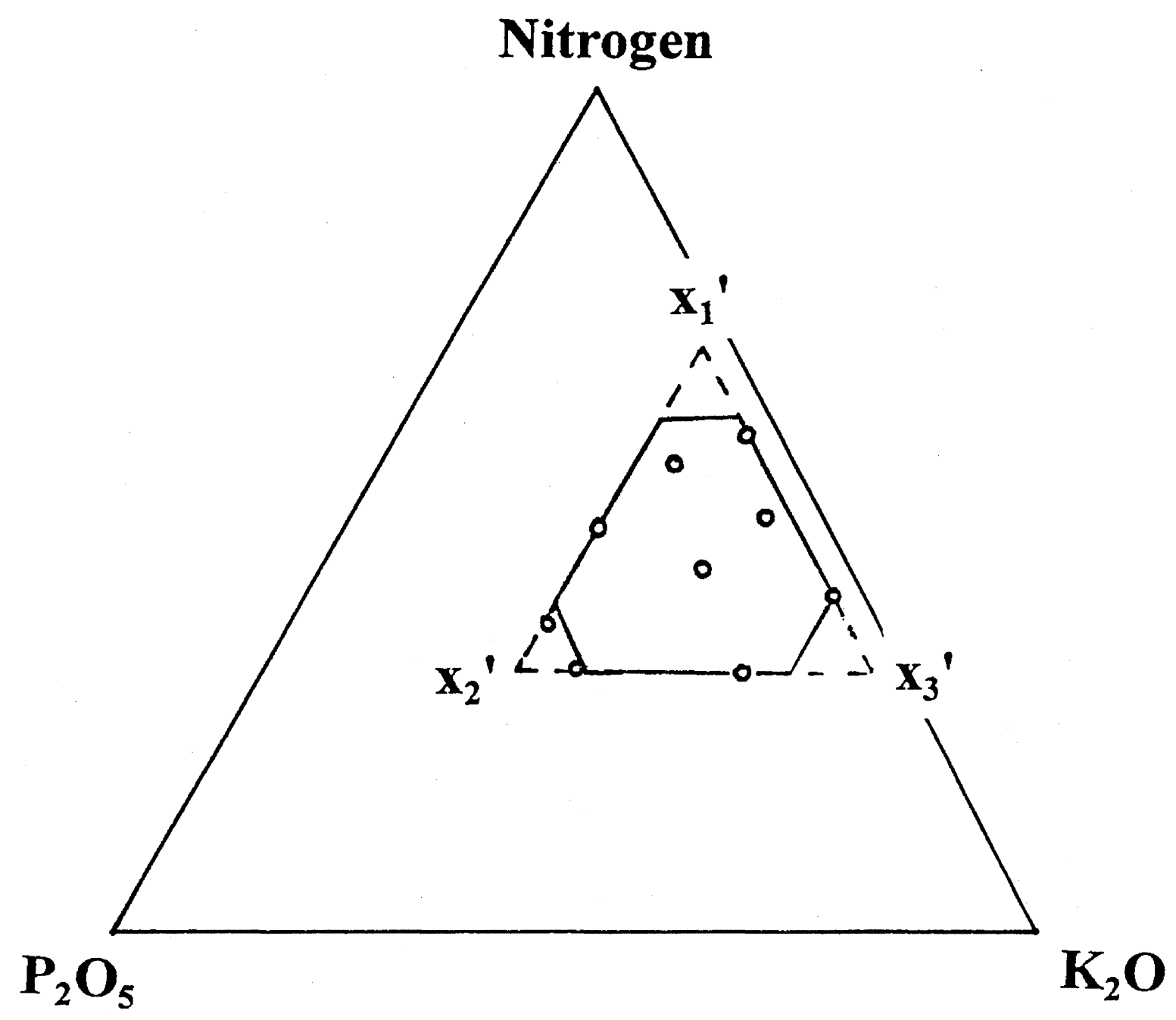

Figure 3. The constrained region in the L-pseudocomponents $x_{1}^{\prime}, x_{2}^{\prime}$ and $x_{3}^{\prime}$. 

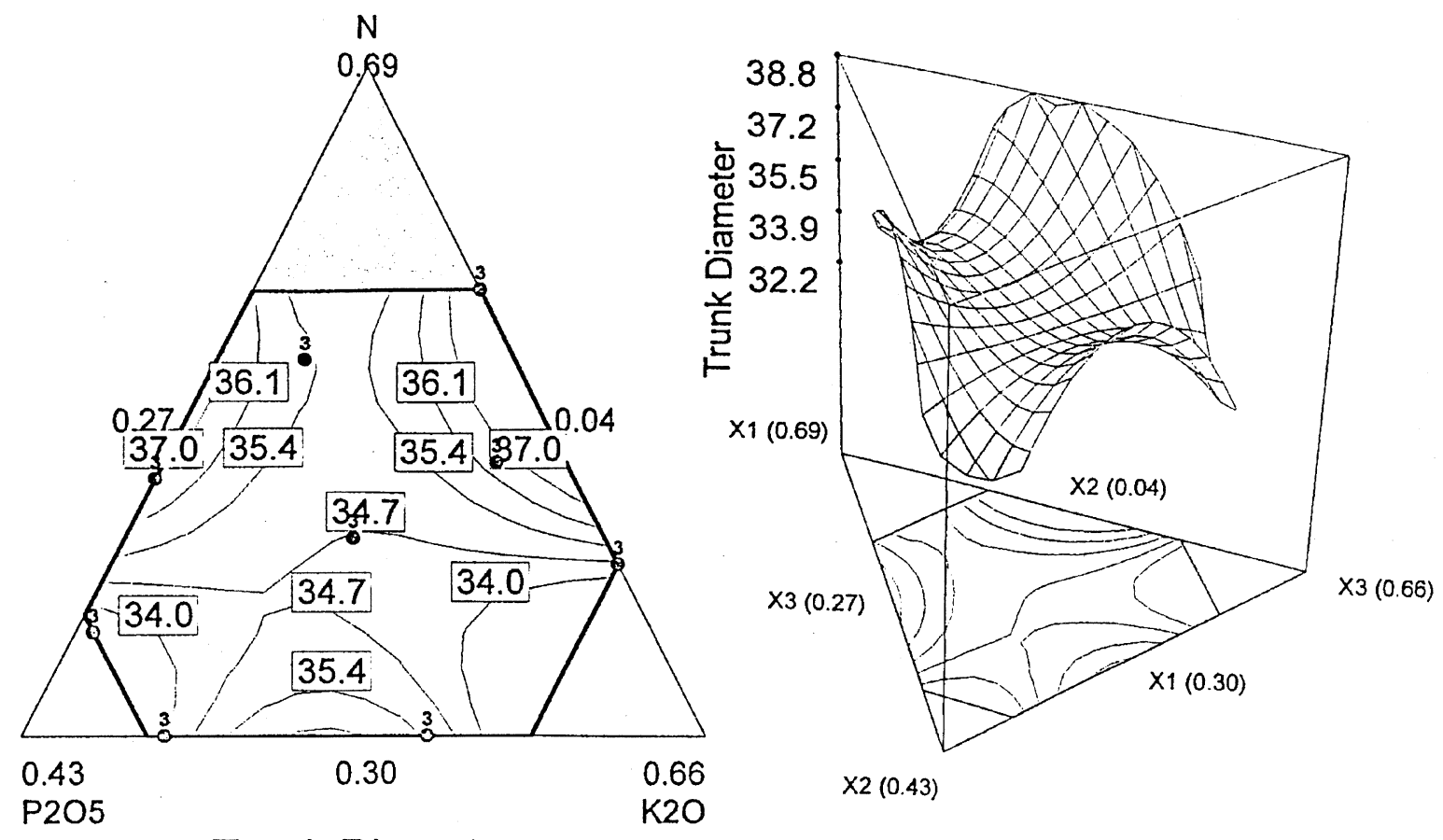

Trunk Diameter

Figure 4. Contour and 3-dimensional plots of the estimated trunk diameter surface averaged over the three rates of fertilization.

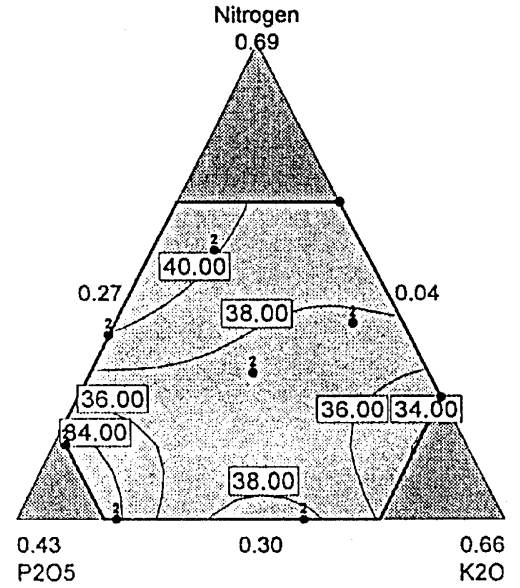

Trunk Dia.-L

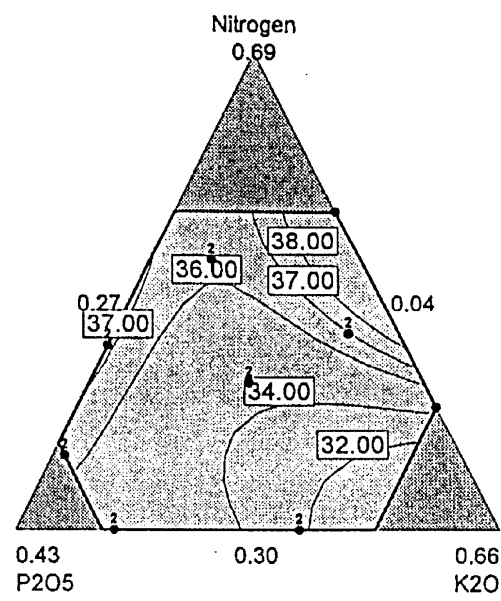

Trunk Dia.-M

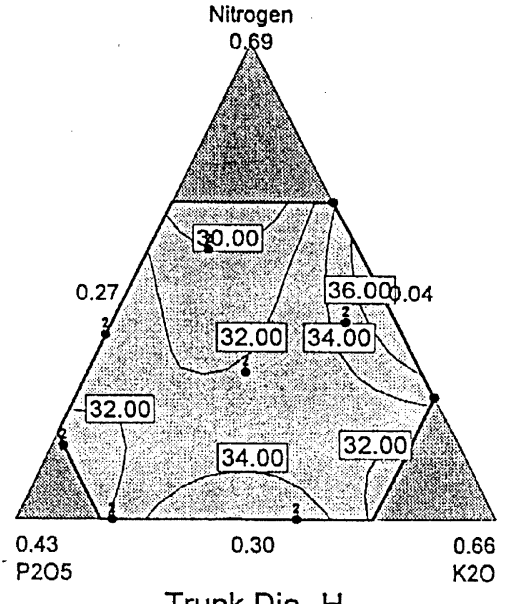

Trunk Dia.-H

Figure 5. Contour plots of the estimated trunk diameter surfaces over the L-pseudocomponent triangle at the low, medium, and high rates. 